\title{
Filtering of LiDAR Point Cloud Data Based on new TIN Algorithm
}

\author{
Lichun SUI ${ }^{1, a}$, Jianfeng $\mathrm{ZHU}^{2, \mathrm{~b}}$, Haixiong $\mathrm{ZHU}^{3, \mathrm{c}}$, Mianqing $\mathrm{ZHONG}{ }^{4, \mathrm{~d}}$ \\ College of Geology Engineering and Geomatics, ChangAn University, No.126 Yanta Road, Xi'an \\ 710054, China
}

National Administration of Surveying, Mapping and Geoinformation engineering research center of Geographic National Conditions Monitoring, Xi'an 710054, China

College of Geology Engineering and Geomatics, ChangAn University, No.126 Yanta Road, Xi'an 710054, China

aemail: 524138055@qq.com, bemail: 710778177@qq.com, cemail: 951755015@qq.com

Keywords: LiDAR; point cloud; improved TIN; ground points; filtering; morphology

Abstract: In this paper, the new method is proposed for filtering of airborne LiDAR data based on improved Triangulated Irregular Network(TIN) algorithm and the details of filter principle is described. Firstly, LiDAR point cloud data is organized and designed by regular grid and TIN, the seed points from point cloud data are selected by regional sub-block method or mathematical morphology. Then, an initial sparse TIN is created from the seed points and densified upward gradually and the ground points are extracted through an interactive process. In experiments it is shown that this filtering method can effectively remove different sizes of buildings, low vegetation and other objects, and keep topographical features better.

\section{Introduction}

Due to the diversity of the terrain and the complexity of the surface features, LiDAR point cloud filtering is still in the stage of research and development with many difficulties and remains a focus of the research. According to Zhang[1], the algorithms of filtering can be roughly divided into these kinds: Morphological method[2], moving window method[3], iterative least-squares interpolation method[4], slope based filtering[5], TIN(Triangulated Irregular Network) based filtering [6,8] and other methods.

On the TIN based filtering, Axelsson[6] assumes that the local topography is flat, firstly select the lowest point within the local area as the seed point to build the initial sparse TIN, then densify the TIN in an iterative process through adapting to the points from below to above by the distance and angle to the triangle.Sohn[7] proposed another idea. He establishes a TIN tetrahedron at first, then gets the ground points by refining the model downward and upward with the Minimum Description Length (MDL) criterion. Haugerud[8] builds the TIN model using the original point cloud data directly, and then calculate the curvature parameters from this TIN model. The point whose curvature changes rapidly is regarded as the non-ground point.

Depend on the TIN based algorithms introduced above, this paper proposes a new method for filtering LiDAR point cloud data based on progressive TIN. This method use grid and TIN as the organization format for LiDAR point cloud data. Firstly, block the whole dataset depend on the global terrain, and seed points are selected by regional sub-block method or mathematical morphology. Then, an initial sparse TIN will be created from these seed points. Ground points will be extracted in an interactive process and the TIN will be densified at the same time. The experiments show that this filtering method can effectively removes different sizes of buildings, low 
vegetation and other objects, but the topographical features will be kept very well.

\section{Principle of filtering based on progressive TIN}

As shown in figure $1, P$ is a discrete point, $V_{1} 、 V_{2} 、 V_{3}$ is three vertex of the triangle, $d$ is the distance from point $P$ to triangle plane, $O$ is the foot point, $\alpha_{1}, \alpha_{2}, \alpha_{3}$ are angles formed by point $P$, three triangle vertex and foot point. Defined $\alpha_{1}, \alpha_{2}, \alpha_{3}$ as the iterative angle, and defined $d$ as the iterative distance. The progressive TIN filtering method takes the iterative angle and the iterative distance as filter parameters, and extracts new ground points by iteratively adjusting the TIN model upward.

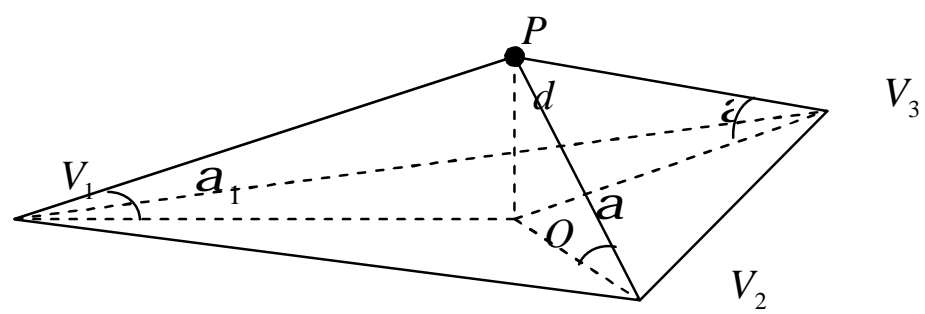

Fig.1 Parameters Illustration in Progressive TIN Filtering Algorithm

Mathematically, the equation of the plane determined by three vertices of the triangle is:

$$
A x+B y+C z+D=0 \text {. }
$$

The distance between point $P$ and triangle vertices $V_{1}, V_{2}, V_{3}$ :

$$
S_{i p}=\sqrt{\left(X_{v i}-X_{p}\right)^{2}+\left(Y_{v i}-Y_{p}\right)^{2}+\left(Z_{v i}-Z_{p}\right)^{2}}(i=1,2,3) .
$$

The iterative distance $d$ and iterative angle $\alpha$ can be expressed as:

$$
\begin{gathered}
d=\left|\frac{A x_{p}+B y_{p}+C z_{p}+D}{\sqrt{A^{2}+B^{2}+C^{2}}}\right| . \\
\alpha_{i}=\arcsin \frac{d}{S_{i p}}(i=1,2,3) .
\end{gathered}
$$

The iterative angle and iterative distance reflect the degree of TIN upward lift. The smaller the iterative angle is, the less possible will low vegetation be mistakenly classified to ground points. Iterative distance ensures that the triangulation will not take a big jump when the triangle is very large, in particular, some low narrow buildings can be effectively filtered out. Just as showed in Figure 2, $\mathrm{AB}$ is a low narrow building, $\mathrm{C}$ is a triangle vertice. The iterative angle formed by the point $\mathrm{C}$ and the middle point of the building may be within the threshold, but because of iterative distance limitation, the middle point of the building will not be added to the TIN model. 


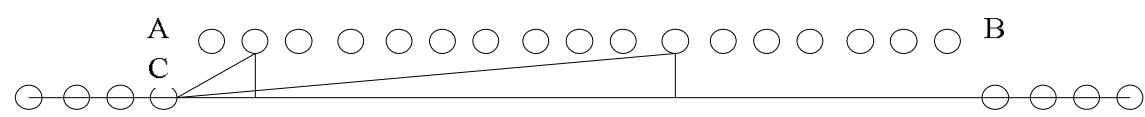

Fig.2 Illustration of Iteration Distance

\section{Filtering experiment}

\section{Test data}

This experiment uses two dataset with typical terrain,to test the validity and reliability of this algorithm.Test data 1 is a mixed terrain with dense forests, vegetation, sharp slope and buildings. The data area is $1100 \times 1100 \mathrm{~m}$, the maximum height difference is about 40 meters, the average distance between the points is approximately 3.2 meters, and the total number of discrete points is 86419.Test data 2 is the standard data recommended by ISPRS.It has 17,875 points[9]. The test data contains different size of buildings, trees, vegetation and roads.

\section{Results and analysis}

Figure 3 (a) shows the point cloud of Test Data 1 before filtering. Figure 3 (b) shows the point cloud after filtering. From Figure 3 (b) we can see that the overall performance of this filtering algorithm is good. It is effective for a variety of ground targets. Different sizes of buildings, vegetation (or low vegetation) and other objects can be effectively filtered out. The abnormal points such as the empty region points are also taken into account and the terrain features can be well maintained.

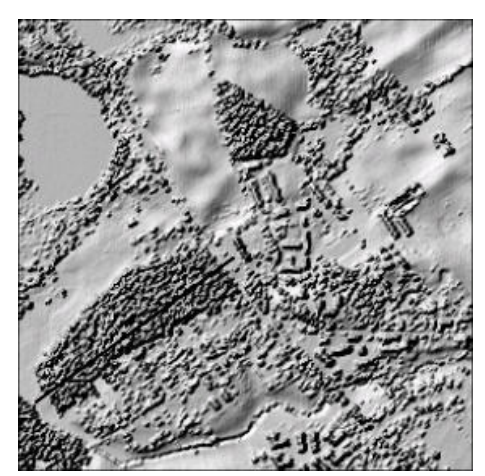

(a) Shaded relief maps of raw data

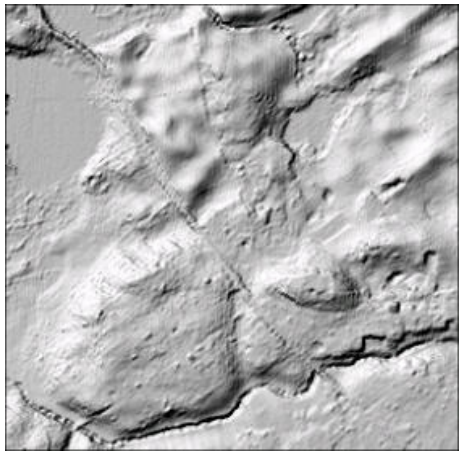

(b) Shaded relief maps of filtered data

Fig. 3 Result of Test Data 1

Figure 4 (a) shows the original standard data sample 51 provided by ISPRS. Figure 4 (c) shows the filtering result. Through comparing the point cloud before and after filtering, it can be concluded that the basic features of the terrain have been maintained after filtering. 


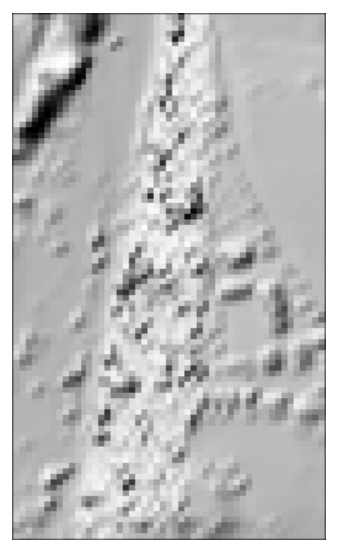

(a) Raw data

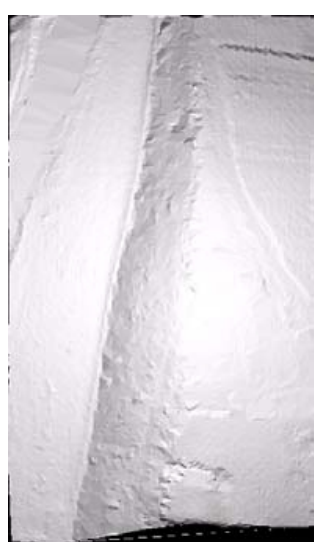

(b) ISPRS result

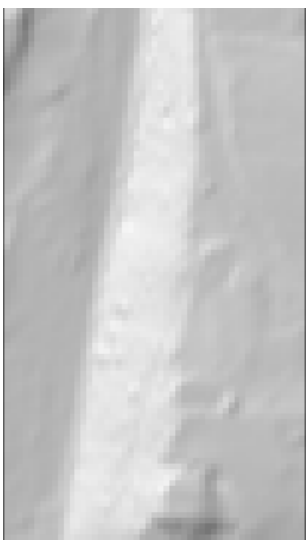

(c) Our result

Fig.4 Result of Test Data 2

Analyze test data 1 and 2 quantitatively using the method recommended by ISPRS. Obtain the parameters $a, b, c$ and $d$ by the interactive statistic using the method mentioned above and then calculate Type I error and Type II error. The statistical results are shown in Table 1. For Test Data 2, can be seen from Table 1, the Type I error of this method is $0.13 \%$ bigger than Axelsson's method, but smaller than other methods. The Type II error of this method is $1.93 \%$ bigger than Pfeifer's method, but smaller than other methods. The results of Test Data 1 are basically in line with this conclusion.

Table.1 Percentage of Type I and Type II Errors

\begin{tabular}{clllllll}
\hline \multirow{2}{*}{ Data } & \multicolumn{3}{c}{ Parameter } & \multicolumn{3}{c}{ error (\%) } \\
\cline { 2 - 8 } & $\mathrm{a}$ & $\mathrm{b}$ & $\mathrm{c}$ & $\mathrm{d}$ & $\mathrm{I}$ & $\mathrm{II}$ & $\mathrm{III}$ \\
\hline 1 & 69638 & 647 & 673 & 15461 & 0.9 & 4.2 & 1.5 \\
\hline 2 & 13902 & 70 & 103 & 3770 & 0.5 & 2.7 & 1.0 \\
\hline
\end{tabular}

\section{Conclusion}

The filtering method proposed in this article process the original LiDAR point cloud without interpolation. It can effectively filter out different sizes of buildings, low vegetation and other objects. It rarely lose ground points, that means Typelerror is small. The accuracy is high so that it can keep the terrain features very well.For large, dense low vegetation and low vegetation on the slopes, the algorithm may regards the low vegetation points as the ground points and this will bring errors. Filtering effect for sharp slope should also be further improved. Dynamically adjusting the iterative angle and distance threshold will be considered in the future research to improve the algorithm's adaptive capacity.

\section{Acknowledgement}

This work was supported by National Natural Scientific Foundation of China (NSFC) grants (No. 41372330). 


\section{References}

[1] Zhang Xiaohong (2007) Theory and methods of airborne LIDAR surveying technology[M]. WuHan: WuHan University Press.

[2] SUI Lichun (2003) Analysis of laser scanner data by means of digital image processing techniques[M]. Munich, Germany : Publishing House Dr. Hut , ISBN 3-89963-022-X.

[3] Petzold B, Axelsson P (2000) Result of the OEEPE WG on Laser Data Acquisition[J].International Archives of Photogrammetry and Remote Sensing, Amsterdam,33(B3):718-723.

[4] Kraus K, Pfeifer N (1998) Determination of terrain models in wooded areas with Airborne Laser Scanner data[J].ISPRS Journal of Photogrammetry \& Remote Sensing,53(4):193-203.

[5] Vosselman G (2000) Slope based filtering of Laser Altimetry data[J]. International Archives of the Photogrammetry, Remote Sensing and Spatial Information Sciences,33(B3):935-942.

[6] Axelsson P (2000) DEM generation from Laser Scanner data using adaptive TIN Models[J]. International Archives of the Photogrammetry, Remote Sensing and Spatial Information Sciences, XXXIII(Pt.B4/1):110-117.

[7] Sohn G, Dowman I (2002) Terrain surface reconstruction by the use of tetrahedron model with the MDL criterion[J].International Archives of the Photogrammetry, Remote Sensing and Spatial Information Sciences,34(A3):336-344.

[8] Haugerud R, Harding A (2001) Some Algorithms for Virtual Deforestation(VDF) of LIDAR Topographic Survey Data[J]. International Archives of the Photogrammetry, Remote Sensing and Spatial Information Sciences, 34(W4):211-218. 